\title{
Cryptocurrency, Artificial Intelligence and Basic Income as Innovative Technological System
}

\author{
Lyubomyr Sopilnyk ${ }^{1}$, Andriy Shevchuk ${ }^{2}$, Vasyl Kopytko ${ }^{3}$
}

${ }^{1}$ Lviv University of Business and Law

99 Kulparkivska Street, Lviv, 79021, Ukraine

${ }^{2}$ Cherkasy State Technological University

460 Shevchenko Boulevard, Cherkasy, 18006, Ukraine

${ }^{3}$ Lviv Branch of Dnipropetrovsk National University of Railway Transport named after Academician V. Lazaryan

12a I. Blazhkevych Street, Lviv, 79052, Ukraine

DOI: $10.22178 /$ pos.37-6

JEL Classification: G00

Received 25.07.2018

Accepted 10.08.2018

Published online 31.08.2018

Corresponding Author:

Lyubomyr Sopilnyk

sopilnyk01@gmail.com

(C) 2018 The Authors. This article

is licensed under a Creative Commons

Attribution 4.0 License @ (1)
Abstract. The common signs of development and its financing of cryptocurrency, artificial intelligence and basic income were determined. The main tendencies and problems of the development of cryptocurrency and artificial intelligence from their appearance to the present time were considered. The combination of cryptocurrency, artificial intelligence and basic income in a unified system of innovative tools for the creation of a new global financial and technological system was substantiated. These three technologies have the features of combining into one innovative system of interaction at different levels from hardware and software to the level of ultimate practical implementation and application in the future. The perception and consideration of these technologies as a single system makes it possible to significantly simplify the approach to their study, design and implementation.

Keywords: cryptocurrency; artificial intelligence; basic income; innovative tools; global financial and technological system.

\section{INTRODUCTION}

The world financial system entered a new phase of innovation transformation using new information technologies with the development of cryptocurrencies. The new digital decentralized system was created during 9 years in the form of a mining, support and maintenance infrastructure with calculations, bidding, placement and creation of new projects. The new innovative system of modernity was created together with cryptocurrency and other technologies, such as artificial intelligence (AI), that will have a decisive influence on the development of the global financial system in the postindustrial and informational economies of the world. Rapid expansion of automation with AI development posed a problem of determining the size and payment of basic income for the population, which will be deprived in the coming years of a large number of workplaces. The combination of the flow of all these processes and their consideration as a single system is relevant to the study, which will allow for the development of common strategies for their evaluation.

\section{LITERATURE REVIEW}

Researchers such as P. Vigna, M. Casey [1], J. Fry, E. Cheah [2], 0. Prymostka [3], and others were engaged in research of cryptocurrencies. The artificial intelligence was investigated by R. Kurzweil [4], S. Russel, P. Norvig [5] and others. The scientific works of A. Atkinson [6], P. Van Parijs [7] and others are devoted to basic income. But most scientists do not consider these three concepts as a system of collaborative interaction that allows them to more effectively study the processes of their development and find practical application. 


\section{RESULTS AND DISCUSSION}

\section{Cryptocurency}

The rapid development of information and digital technologies has led to the natural occurrence of the notion of digital money and digital currency which was implemented with the creation of Bitcoin cryptocurrency and its analogues. The growth of capitalization of major cryptocurrencies, the worldwide spread of technologies such as mining, blockchain and ICO (Initial Coin Offering, like IPO for cryptocurrency market) has forced most developed countries to apply different regulatory methods to a new process, which will now undoubtedly change the global financial system. The study of the possibility of state regulation of these processes is and will be very relevant for most countries of the world in the coming years. A large number of experts see signs of overheating of cryptocurrencies market with the extremely rapid capitalization of it at the end of 2017 , with a significant risk of a crisis and a depreciation of their assets (exchanges, means of property, etc.), which so far have not been met in recent years. There is unpleasant feature of the use of cryptocurrency for governments of the countries, except for the impossibility of direct regulation, is the use of their people close to criminality when anonymity allows them to "launder" money, trade in illicit goods and quickly transfer uncontrollably large sums of money to different parts of the planet. Another problem for the governments is the process of the mining which generate cryptocurrency currencies. Mining consists of searching for specific data sequences rules with minimal hash value on special hardware and software that subsequently used as currencies. Mining has clear signs of financial pyramids with investing real money in equipment and electric power consumption in the obscure rules of pricing of the data. But with rapid increasing exchange rate of Bitcoin mining acquired a mass character and has become popular in many countries. Mining has also become especially popular in Ukraine, as evidenced by the high prices, quick sales and lack of graphics cards, motherboards and ASIC for mining on the Ukrainian market. Now cryptocurrency in Ukraine not adopted any laws, although some miner has been arrested on charges of illegal use of electricity [9]. Some Ukrainian politicians also began to declare their income in Bitcoins that allows them to use these amounts in the future to avoid taxes [10]. The probable path of the evolution of the means of payment for the monetary system during the development of mankind can be depicted in the following figure (Figure 1).

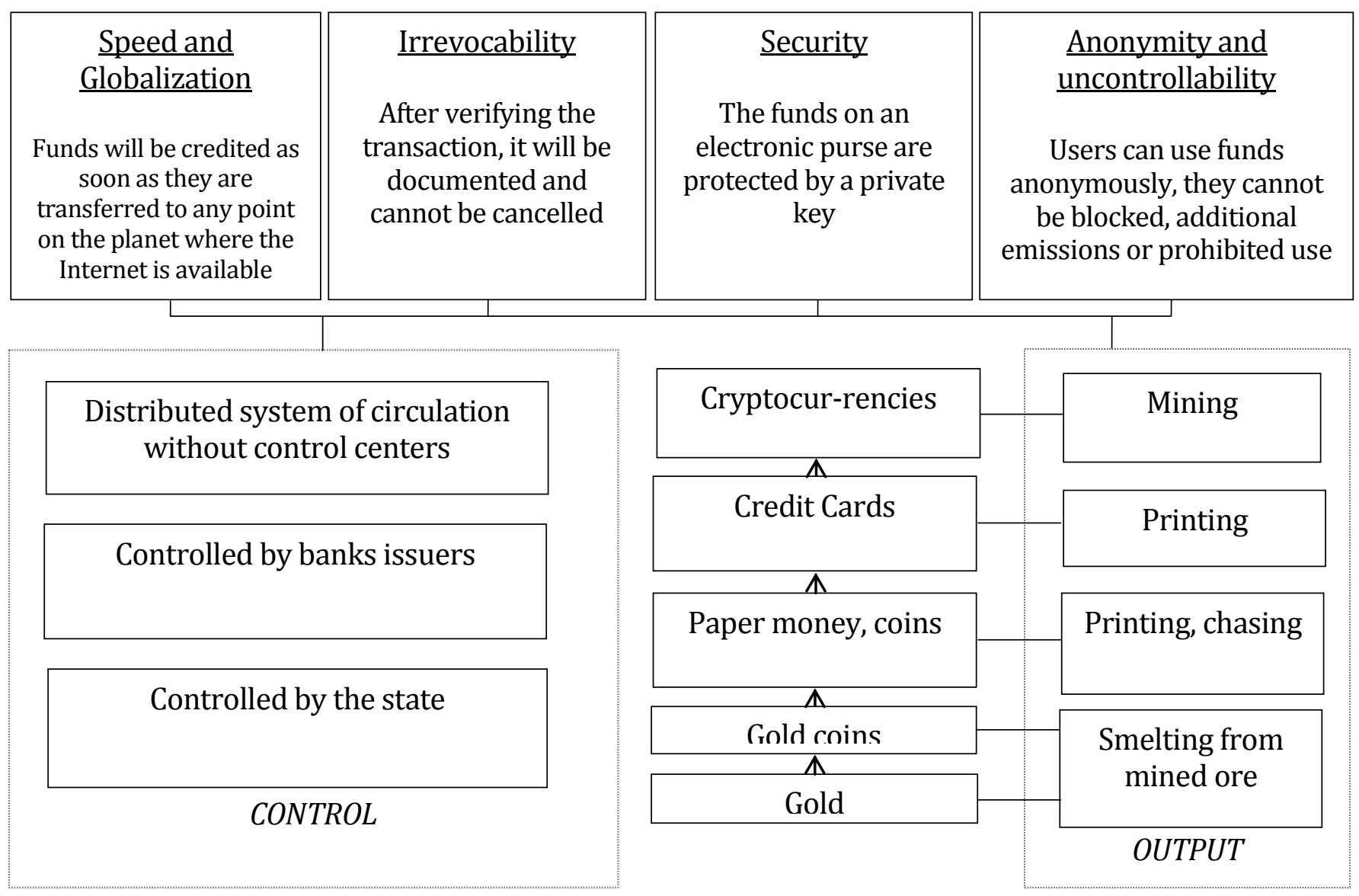

Figure 1 - Evolution of money 
Most countries began with the menacing growth of Bitcoin to try to ban the further development of cybercriminals - restrictive laws passed in China, Russia, the United States. They are trying to detain the owners of electronic exchanges in the US, prohibit the use of ICO (Initial Coin Offering, startups sold to early backers of the project in exchange for legal tender or other cryptocurrencies, but usually for Bitcoin) in China, and outsourcing the property process in other countries. Some governments have been thinking about using cybercriminals and even announcing the creation of their own. Obviously, the need to regulate this process is unquestionable. Government's regulation of cryptocurrency in our opinion should take place in three dimensions: - Take advantage of cryptocurrency goods, and to use them in their financial system, attract investments and prevent the state from lagging behind in a very important process of transforming the world financial system; Prevent the use of cryptocurrency in illegal operations, money laundering, trade in prohibited goods and services; Regulation of the process of investing of citizen's money in technology related to cryptocurrency lures (mining, ICO, etc.) to prevent crises and impairment of invested assets.

A clear position of the countries governments at this stage in the issue of supporting the innovation component of cryptocurrency and the development of a strategy to prevent possible negative effects from their active use and implementation is extremely important. The main advantages of cryptocurrency against fiat money generated by high demand for them were the speed and globalization of the transfer of funds, documented and irreversible transactions, security, the possibility of anonymity in transactions. The rapid growth of the capitalization of Bitcoin and the main cryptocurrencies will be considered in the figure (Figure 2).

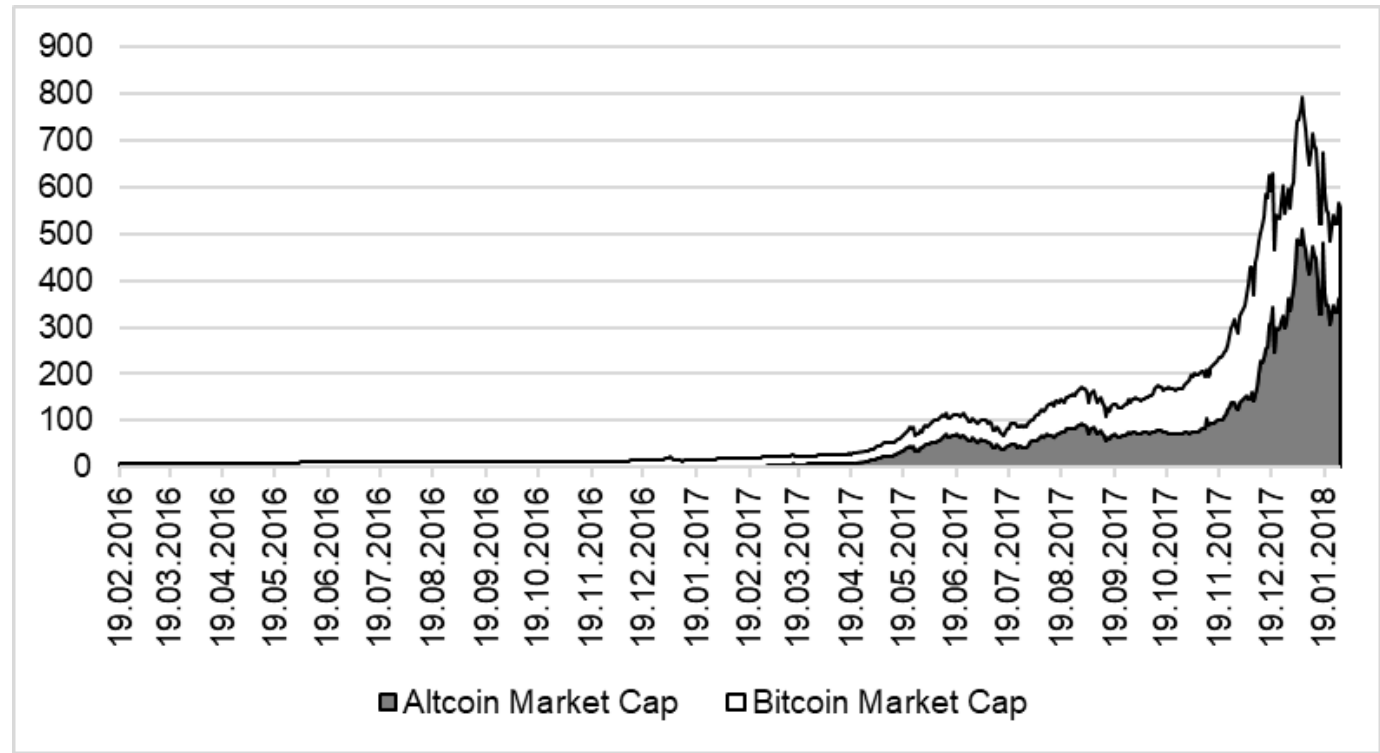

Figure 2 - The capitalization of Bitcoin and other cryptocurrency market in 2016-2018 (US \$ billion) [11]

The figure shows that, since mid-2017, the market capitalization of Bitcoin has increased significantly and reached the maximum value of $800 \mathrm{~B}$ dollars US at the end of the year, after which a decline or correction began. Distribution of the market cryptocurrency in the top ten can be analyzed from Figure 3.

Bitcoin has the first place with an index of $38 \%$ of the total capitalization of cryptocurrency, in the second place coins Ethereum (23\%) and the third Ripple (8\%). Bitcoin has shown over time the use of certain disadvantages, among which the high cost and longtime of the transaction. So can be predicted that its share will continue to fall, at the same time, will increase the share of other cryptocurrency and in the future will be the growth of capitalization of currencies, which will be devoid of major shortcomings and developed with the help of modern digital and network protocols. The Bitcoin has the greatest growth during 2017, at the beginning of 2018, its rate dropped almost doubled from $\$ 20,000$ to $\$$ 11,000 [11]. Leading financiers around the world 
are now raising questions about whether cryptocurrency and Bitcoin, in particular, are a financial pyramid and a bubble that can be devaluated at any time and lead depositors to bankruptcy. Obviously, the new financial system is undergoing repeated transformations and corrections, but in our opinion, impairment is impossible to zero. This is due to the fact that the crypto coins unambiguously found their scope of application in the form of rapid and safe transfer of funds from one point of the world to another, anonymous and documented transactions. The event of a bankruptcy of a particular crypto project will inevitably be redeemed for future use, and thus the complete collapse of the new system is unlikely.
A bankruptcy of some crypto project will lead to the fact that it will inevitably be redeemed for further use, and thus the complete collapse of the new system is very unlikely. It is important to understand that without further development of a new financial system based on new technologies, everything can quickly lose its growth rate. The new financial instrument with the use of cryptocurrency and blockchain technology has actually been created. It is even backed up with hardware: computing power for mining and transaction support. The sphere of direct use of the most crypto of money and the products and services that can be purchased for them is not formed.

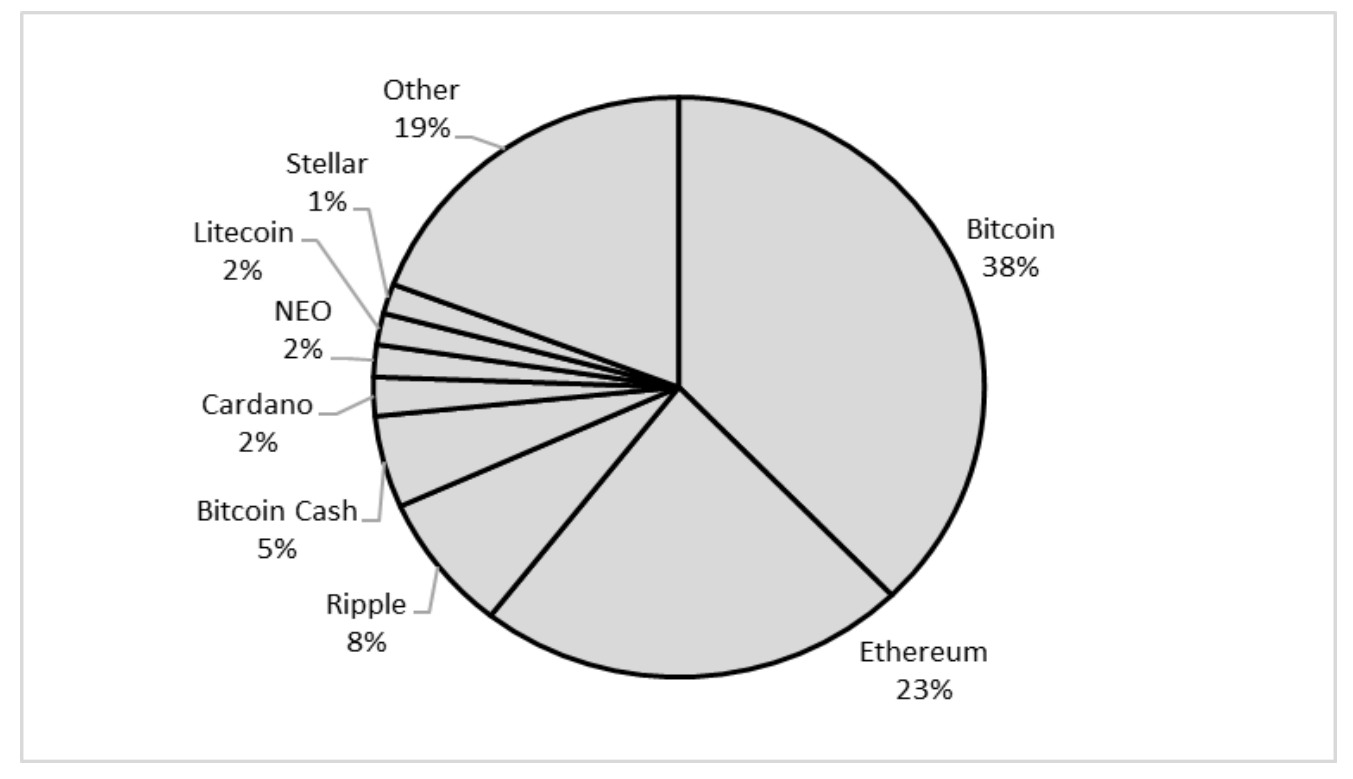

Figure 3 - Capitalization of Bitcoin and other cryptocurrency by the beginning of 2018 (\%) [11]

It should also be noted here that the present research may be aimed at developing a theoretical and methodological approach to the diagnosis of the system of interaction between the government and business in terms of public eprocurement [14].

\section{Artificial Intelligence and basic income}

Obviously, the next step will be the formation of the market of goods and services, where the main means of payment will be not fiat money, but digital. In our opinion, the main product in such a market in the future will be artificial intelligence. This is due to several reasons: the rate of investments in artificial intelligence correlates with the volumes of money invested in crypto- currency; the computing power created in the form of trusts for property financing, for products for fast calculations using CPU and GPU processors, the increased speed of distributed computing through the Internet becomes a large computing power, the only real means for which the use of which remains the training of neural networks for artificial intelligence; these two new innovative tools (cryptocurrency and AI) are very closely interconnected, which determines their mutual use in the future. Another important instrument of the new innovation system that is currently being formed in our opinion should be the basic income [6], [7]. There are now well founded reasons to fear the rapid development of artificial intelligence in the world which will eliminate the work of a large number of people through the automation of simple monotonous 
labor. Among the examples of what has already been practically done is the development of the autopilot program for cars in the United States and the fear that a large number of truck drivers will lose their jobs through this innovation. Thus, compensatory mechanisms must be developed that smooth out significant transformations in the areas of human activity with the development of AI. This compensatory mechanism in re- lation to employees who lose their jobs should become the basic income, the volume of which in the future should determine and regulate by the AI on the basis of constant accumulation and processing of data. So as for reliable data processing on financial operations will require technology blockchain or similar, then obviously paid the basic income will be crippled (Figure 4).

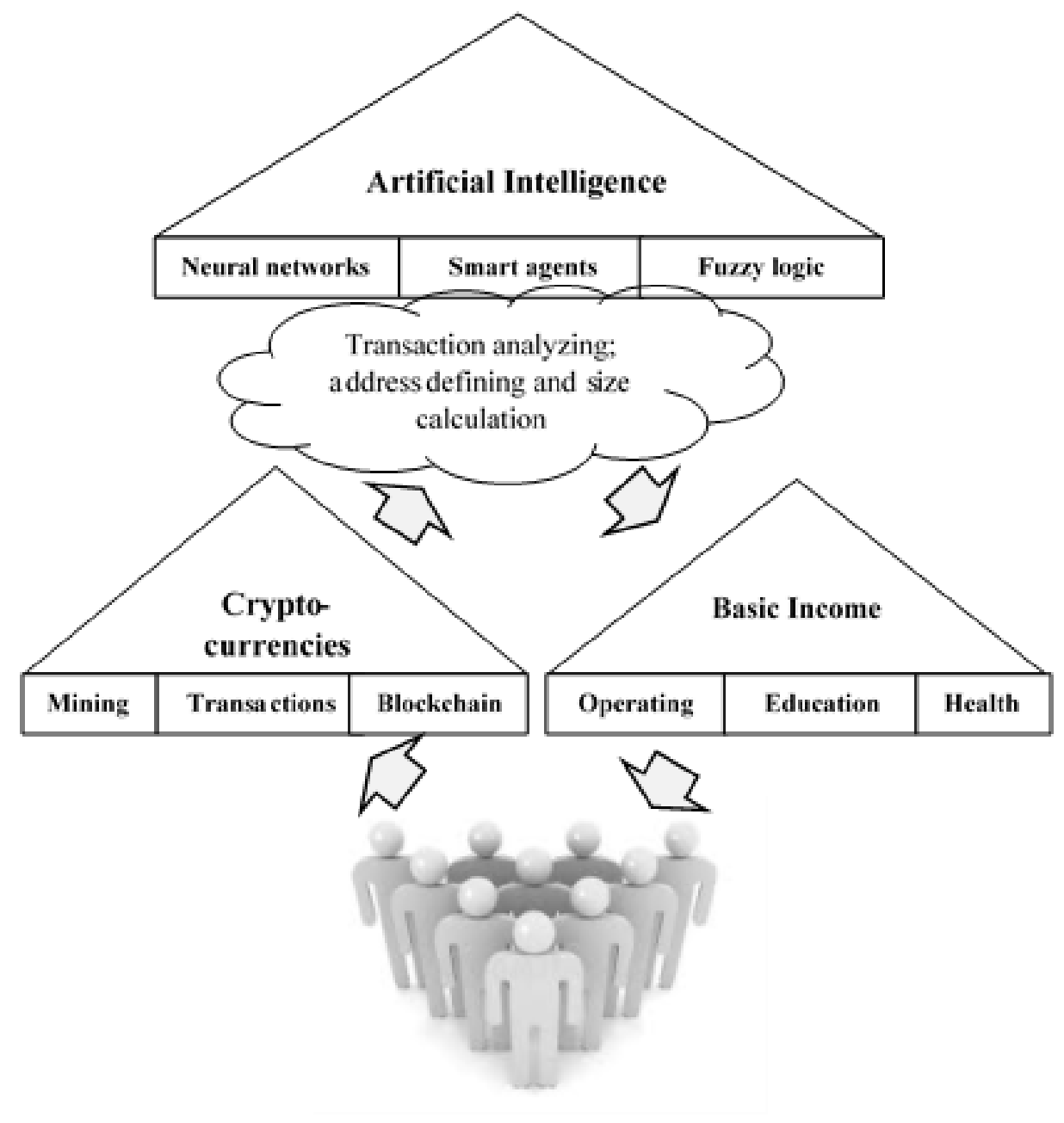

Figure 4 - Al, cryptocurrencies, basic income and human interaction diagram

The defined scenario in the diagram is as follows: AI determines the size and address of the basic income payment using the algorithms of neural networks, smart agents and fuzzy logic. Payments are made in cryptocurrency and recorded further expenses by the population. Amendments are made according to the money spent by people on the priorities of self-improvement, training and health improvement in the next steps. Developed societies for now were not ready to enter the basic income and voted against on the referendum in Switzerland but it is unknown whether the choice will remain in case of transformations of the whole branches of activity and the loss of employment of a large number of workers in it.

Thus, now the whole direction of economic activity is formed, the new industry is based on innovative transformations of the existing financial system in the form of application of cryptocurrency and related technologies. Crypto projects create a new sphere of economic activity along with other related technologies where the product is the digital means of exchange analogous 
fiat money, means of manufacturing equipment for the property and service of transactions. New technologies in the new system are also used in the form of blockchain, new protocols of interaction between equipment and users of the Internet that create the sphere of consumers. Significant investment in projects in 2017 made it possible to develop and improve the software and hardware necessary for the functioning of the industry, which has very large prospects for the transition to a new level of its use for creating a software base of AI. Our assumption is partly confirmed by the necessity of having computing power for the development and operation of AI, which have already been developed and improved due to the development of cryptocurrency with hardware and software for its functioning. Otherwise, the question of the size of the basic income and the addressing of it may not be trivial and require certain calculations and corrections, and all stages of payment, which will require AI. Therefore, it is natural to assume that all these elements are in a certain interconnection and at the stage of development and formation in the modern global financial system.

\section{CONCLUSIONS}

Consequently, the global financial and economic system is currently in the process of global transformation, which is associated with the development of new technologies in the form of crypto-currency, AI and basic income. We found that all these three technologies have the features of combining into one innovative system of interaction at different levels from hardware and software to the level of ultimate practical implementation and application in the future. This is evidenced by the pace of development and financing of cryptocurrency and IT technologies, the direct link between these technologies in terms of using joint tools and implementation and their direct assignment. The perception and consideration of these technologies as a single system will make it possible to significantly simplify the approach to their study, design and implementation. Further research should be aimed at developing a theoretical and methodological approach to modeling processes of the transformation of the global financial and technical system with the help of cryptocurrency, AI and basic income.

\section{REFERENCES}

1. Vigna, P., \& Casey, M. (2015). The Age of Cryptocurrency: How Bitcoin and Digital Money Are Challenging the Global Economic Order. New York: St. Martin's Press.

2. Fry, J., \& Cheah, E.-T. (2016). Negative bubbles and shocks in cryptocurrency markets. International Review of Financial Analysis, 47, 343-352. doi: 10.1016/j.irfa.2016.02.008

3. Prymostka, 0. 0. (2016). Problems and perspectives of institutional market regulation of cryptocurrency. Ekonomika. Finansy. Menedzhment, 5, 69-79.

4. Kurzweil, R. (2016). The singularity is near: when humans transcend biology. London: Duckworth.

5. Russell, S., \& Norvig, P. (2003). Artificial Intelligence: A Modern Approach (2nd ed.). Upper Saddle River: Pearson.

6. Atkinson, A. B. (2004). Public economics in action: The basic income/flat tax proposal. Oxford: Clarendon Press.

7. Van Parijs, P. (2004). Basic Income: A Simple and Powerful Idea for the Twenty-First Century. Politics \& Society, 32(1), 7-39. doi: 10.1177/0032329203261095

8. CoinMarketCap. (2018). Top 100 CryptoCurrencies by Market Capitalization. Retrieved July 1, 2018, from https://coinmarketcap.com

9. Helms, K. (2017, August 14). Arrests of Bitcoin Miners in Ukraine Spark Questions About Legality. Retrieved from https://news.bitcoin.com/arrests-bitcoin-miners-ukraine-questions-legality

10. Durden, T. (2017, August 16). Ukrainian Lawmakers Disclose $\$ 45$ Million in Bitcoin Holdings. Retrieved from https://www.zerohedge.com/news/2017-08-15/ukrainian-lawmakers-disclose45-million-bitcoin-holdings

11. Coin Dance. (2018). Bitcoin Statistics. Retrieved July 1, 2018, from https://coin.dance/stats 
12. Shevchuk, A. V. (2016). Artificial Intelligence and Intellectualization: New Prospects for Economic Development. Scientific Review, 4(25), 27-36.

13. Venture Scanner. (2018). Interest in AI Startups Exploded in 2017. Retrieved July 1, 2018, from https://www.venturescanner.com/blog/2018/interest-in-ai-startups-exploded-in-2017

14. Yuzevych, V., Klyuvak, O., \& Skrynkovskyy, R. (2016). Diagnostics of the system of interaction between the government and business in terms of public e-procurement. Economic Annals-XXI, 160(7-8), 39-44. doi: 10.21003/ea.v160-08 